\title{
What are the implications of the spontaneous spleno-renal shunts in liver cirrhosis?
}

\author{
Giovanni Tarantino*1, Vincenzo Citro ${ }^{2}$, Paolo Conca ${ }^{1}$, Antonio Riccio'1, \\ Marianna Tarantino ${ }^{3}$, Domenico Capone ${ }^{4}$, Michele Cirillo ${ }^{5}$, \\ Roberto Lobello ${ }^{5}$ and Vittorio Iaccarino ${ }^{3}$
}

\begin{abstract}
Address: ${ }^{1}$ Department of Clinical and Experimental Medicine, Federico II University Medical School of Naples, Naples, Italy, ${ }^{2} \mathrm{Hepatology}$ Unit of General Medicine, "Mauro Scarlato" Hospital, Scafati, ASL SA/1, Scafati, Italy, ${ }^{3}$ Department of Biomorphological and Functional Sciences, Federico II University Medical School of Naples, Naples, Italy, ${ }^{4}$ Department of Neurosciences, Unit of Clinical Pharmacology, Federico II University Medical School of Naples, Naples, Italy and ${ }^{5}$ Department of Oncology and Endocrinology, Gastrointestinal Surgery Unit, Federico II University Medical School of Naples, Naples, Italy

Email: Giovanni Tarantino* - tarantin@unina.it; Vincenzo Citro - v.citro@libero.it; Paolo Conca - paolo.conca@unina.it; Antonio Riccio - antonio.riccio@unina.it; Marianna Tarantino - giovanni.tarantino@unina.it; Domenico Capone - docapone@unina.it; Michele Cirillo - michele.cirillo@unina.it; Roberto Lobello - roberto.lobello@unina.it; Vittorio Iaccarino - vittorio.iaccarino@unina.it

* Corresponding author
\end{abstract}

Published: 24 November 2009

BMC Gastroenterology 2009, 9:89 doi:10.1186/147/-230X-9-89
Received: 7 August 2009

Accepted: 24 November 2009

This article is available from: http://www.biomedcentral.com/I47/-230X/9/89

(c) 2009 Tarantino et al; licensee BioMed Central Ltd.

This is an Open Access article distributed under the terms of the Creative Commons Attribution License (http://creativecommons.org/licenses/by/2.0), which permits unrestricted use, distribution, and reproduction in any medium, provided the original work is properly cited.

\begin{abstract}
Background: Although significant advances are expected to be made in the assessment of the portal hypertension-related complications, the prognostic role of spleno-renal shunts has not been fully explored so far. Clarifying this aspect could help tackle the life-treating events occurring in patients suffering from liver cirrhosis. The aim of the study was to analyze the relationships between the spleno-renal shunts presence at doppler ultrasound and the liver cirrhosis complications.

Methods: Design: eighty one patients out of 129 formed the study population (35 females). Chronic liver damage in these patients was caused by HCV (66), HBV (2), alcohol abuse (2) or unknown etiology, likely non-alcoholic steatohepatitis (II). Setting: two Liver Units of university/primary hospitals in Southern Italy. Main outcome measures: grading of esofageal varices; detection of ascites: assessment of hepatic encephalopathy; evaluation of liver cirrhosis severity; tracking hepatocellular carcinoma; doppler features of spleno-renal shunts and splenic flow velocity; spleen longitudinal diameter at sonography.

Results: The prevalence of spleno-renal shunts was 18.5\%, without no difference concerning the etiology (HCV versus non$\mathrm{HCV}, \mathrm{p}=0.870$ ); the prevalence of hepatocellular carcinoma in patients with spleno-renal shunts was superior to that of patients without them (Pearson Chi-square, $p=0.006$, power of sample size $74 \%$ ), also after adjustment for liver decompensation $(p=$ 0.024). The median score of hepatic encephalopathy in patients with and without spleno-renal shunts was similar, i.e., 0 (range, $0-2$ ) versus $0(0-3), p=0.67$. The median splenic vein flow velocity in patients with spleno-renal shunts was significantly inferior to that of patients without them, i.e., $13 \mathrm{~cm} / \mathrm{sec}(95 \%$ confidence intervals, $6-18)$ versus $21 \mathrm{~cm} / \mathrm{sec}(17-24), \mathrm{p}<0.000 \mathrm{I}$. By far the largest percentage of large esophageal varices was in patients without spleno-renal shunts $(p=0.005)$. In contrast, the frequency of ascites and hepatic encephalopathy severity was overlapping in the two groups. BMI values but not Child-Pugh's classification predicted spleno-renal shunts (Ors $=1.84,95 \%$ confidence intervals $=1.28-2.64, \mathrm{p}=0.00 \mathrm{I}$ and $1.145,95 \%$ confidence intervals $=0.77-1.51, p=0.66$ ).
\end{abstract}

Conclusion: Taking into consideration the relatively small sample size, patients with spleno-renal shunts are burdened by an increased incidence of hepatocellular carcinoma. BMI predicted the spleno-renal shunts presence. 


\section{Background}

The understanding of mechanisms regulating Portal Hypertension ( $\mathrm{PH}$ ) has been incomplete so far, although researchers have struggled for years. $\mathrm{PH}$ is primarily caused by an increase in resistance to portal outflow and secondly by a growth in splanchnic blood flow [1]. In a later phase, these changes lead to a hyperkinetic circulation that raises cardiac output and reduces systemic vascular resistance as well as perfusion pressure. Regional alterations in vasoreactivity (vasodilation and vasoconstriction), sinusoidal remodelling and capillarization, angiogenesis, venous thrombosis, and obviously vascular distortion, all play a certain role in the pathophysiology of PH by contributing to circulatory impairment and expansion of the collateral circulation. Among vasoactive substances activated in $\mathrm{PH}$, nitric oxide is considered as the most important vasodilator. Endothelin-1 and cyclooxygenase-derived prostaglandins are the foremost vasoconstrictor factors, apart from the sympathetic overactivity. Furthermore, a major role has been attributed to activated hypercontractile hepatic stellate cells, which cause vascular remodelling as an adaptive response to the changed balance in vasoactive substances. It has recently demonstrated that endothelial dysfunction is determinant in $\mathrm{PH}$ development. Finally, an increase in the splanchnic vascular bed size mediated by a Vascular Endothelial Growth Factor (VEGF)-dependent angiogenic process has been claimed to significantly contribute to increased overall blood flow in splanchnic tissues of PH animals. In addition, this VEGF-dependent angiogenesis also plays a crucial role in the formation of portal-systemic collateral vessels, which include Spleno-Renal shunts (SRS [2]). Notably, novel research has highlighted that $\mathrm{PH}$ is an independent predictor of HepatoCellular Carcinoma (HCC) development [3]. Beyond the formation of portosystemic shunts, of which Esophageal Varices (EV) have the greatest clinical impact and the most severe complications, PH leads to Hepatic Encephalopathy (HE [4]) and ascites [5]. Other manifestations of $\mathrm{PH}$ include portal hypertensive gastropathy [6] and Large Spontaneous Shunts (LSS [7]). LSS refer to the presence of patent paraumbilical vein, SRS, ano-rectosigmoid varices with or without portal hypertensive colopathy. The prevalence of umbilical vein patency ranges from $6 \%$ to $30 \%$ in patients with PH [8]. SRS are present in cirrhotics from nearly $14 \%$ up to $21 \%[9,10]$. Rectosigmoid varices are present in nearly one third of cirrhotic patients, $4 \%$ of whom have a lower gastrointestinal hemorrhage [11]. LSS, the presence of which was assessed by portal venous phase multidetector-row spiral computed tomography, have been shown to be responsible for recurrent or persistent HE [12]. In patients with liver cirrhosis, with and without bleeding varices, many imaging techniques are available to detect collaterals, beyond the previously quoted one. In fact, Magnetic Resonance Imaging (MRI [13]), single photon emission computed tomography $[14,15]$, selective intraarterial digital subtraction angiography [16], scintiphotosplenoportography [17], but also the percutaneous transhepatic portography [18], are quite conclusive in diagnosing SRS. Reports published in the literature to date have shown that all patients with hepatic cirrhosis should be studied by using Doppler UltraSound (DUS) techniques [19], being the remainder methods invasive or extremely expensive and then unsuitable in close followups. DUS can provide a great deal of information about the morphology and hemodynamics of $\mathrm{PH}$ and consequently spontaneous shunts can be identified [20]. They may mimic surgically created shunts in their large volume-flow capacity. In contrast, other Authors believe that hepato-splenic hemodynamics, as studied at DUS, are only weakly correlated to portal pressure, suggesting measuring them with MRI [21]. Anyway, among blood flow parameters, Splenic Vein Flow Velocity (SVFV) is considered to be specifically related to the stages of liver damage [22] and to EV bleeding [23]. Although significant advances are expected to be made in the diagnosis and management of the PH-related complications, in the face of an increasing burden of chronic liver disease, the prognostic role of LSS has not been presented in a convincing manner so far. Clarifying this aspect could help correctly approach the ongoing, life-treating events occurring in patients suffering from liver cirrhosis. Therefore, the objective of this study was to evaluate the relation of SRS, detected by an easily reproducible and reliable tool, i.e., DUS, to the severity of ascites, EV, HE and the presence of HCC. Furthermore, we intended to seek any association of these imaging parameters with the degree of liver failure.

\section{Methods}

This research was performed screening 129 consecutive patients with established liver cirrhosis admitted to two Liver Units of university/primary hospitals in Southern Italy from October 2006 to December 2008. The study was carried out according to the principles of the Declaration of Helsinki and an informed written consent was obtained from each patient.

\section{Exclusion criteria}

Out of the initial patients, 23 were excluded because their instrumental examinations (DUS) had been previously performed in different centres. Fourteen patients, who had undergone endoscopic EV ligation therapy, and eight, who had received beta-blockers, were also disallowed from the study because prior treatment might have caused a change in DUS features. Three patients were left out for the detection of thrombosis of the portal vein. 


\section{Inclusion criteria}

The remaining 81 patients formed the study population (35 females) whose age was 68.2, 8.18, 66.4-70 years (mean, +/-SD, 95\% CI). Chronic liver damage in these patients was caused by hepatitis $C(n=66)$, hepatitis $B(n$ $=2)$, alcohol abuse $(\mathrm{n}=2)$ or unknown etiology, likely Non-Alcoholic SteatoHepatitis (NASH) $(n=11)$. Forty seven patients had compensated cirrhosis of the liver. For 71 patients, the diagnosis of cirrhosis was established by contextual clinical (spider nevi, hepato-splenomegaly), laboratory (low serum total cholesterol, prothrombin activity and pseudocholinesterase levels, reduced white blood cell and platelets count, globulin/albumin ratio $>1,[24])$ as well as antecedent imaging data. Being these parameters inadequate to confirm the diagnosis in 10 patients, successively assigned to the Child's class A, a liver biopsy was performed. The non-invasive assessment of liver cirrhosis was blindly performed de novo to all patients by experts on the basis of UltraSound (US) and DUS examinations (coarse echo-texture, nodularity presence, increased caudate/right lobe ratio, hypertrophy of the left lobe, characterized by a rounded inferior marginal edge, and portal vein enlargement with decreased flow velocity, absence of a normal doppler waveform, hepatofugal flow). Alcohol abuse was diagnosed according to the DSM-IV criteria, by means of screening tests such as MAST (Michigan Alcohol Screening Test) and CAGE (Cut down, Annoyed, Guilty, and Eye opener), as well as random tests for blood alcohol concentration and the use of a surrogate marker, e.g., Mean Corpuscular Volume.

Prestudy agreement, shared by all the investigators, gave place to common diagnostic procedures in order to meet the target of concordance. Patients should have fulfilled the following criterion at entry, i.e., US/DUS, endoscopic and laboratory examinations performed within four weeks of each other.

\section{Endoscopy}

EV were graded according to a previous classification, i.e., F1 small and straight; F2 moderately sized, tortuous, and occupying less than one third of the lumen; F3 large, coiled, and occupying one third or more of the lumen [25]. Large EV (LEV) were considered F2 and F3.

\section{Ascites presence at ultrasound}

The superior end of the right paracolic gutter and the pelvis were carefully assessed at US. Small quantities were sought for around the liver or spleen surface and in the Morrison's pouch [26]. The presence of peritoneo-pleural communications was determined. BMI of patients with ascites was adjusted taking into consideration the weight before this complication.

\section{Hepatic encephalopathy grade}

HE was graded based on the level of consciousness, intellectual functions, behaviour and neuromuscular functions according to West Haven (W-H) criteria [27]. W-H grade 0 or minimal encephalopathy was ascertained as previously described [28].

\section{Assessment of liver cirrhosis severity}

In all patients the severity of illness was assessed using a modified Child-Pugh's classification [29], Table 1). The decompesation criterion was set at score 8 of Child-Pugh's classification [30], although some patients presented light ascites or minimal encephalopathy under this cut-off.

\section{Hepatocellular carcinoma detection}

Patients were screened for HCC by performing abdominal US and testing alpha-fetoprotein at admission, in course of regular 6-monthly or annually (by computed tomography) surveillance. When a surveillance feature or test was abnormal (>10.9 ng/mL), a triple-phase imaging (MRI) was recommended for evaluation at recall.

Table I: Child-Pugh classification slightly modified.

\begin{tabular}{lccc}
\hline Clinic and laboratory data & Points for increasing abnormality I & $\mathbf{2}$ & $\mathbf{3}$ \\
\hline HE (grade $\left.{ }^{2}\right)$ & None & SHE $(0), ~ I-2$ & $3-4$ \\
Ascites & None at US & Mild or controlled by diuretics & Present despite diuretics \\
PT $(\%$ of activity) & $>70$ & $70-40$ & $<40$ \\
Albumin $(\mathrm{g} / \mathrm{dL})$ & $>3.5$ & $2.8-3.5$ & $<2.8$ \\
Bilirubin $(\mathrm{mg} / \mathrm{dL})$ & $<2$ & $2-3$ & $>3$ \\
\hline
\end{tabular}

IScoring system: 5-6 points, grade A; 7-9 points, grade B; 10-15 points, grade C. ${ }^{2 H E}$ : hepatic encephalopathy; Grade 0: sub-clinical hepatic encephalopathy, SHE, unravelled by Retain tests A or B; Grade I: anxiety, irritability, depression, impaired concentration, sleep disturbances; Grade 2: disorientation, poor short-term memory, disinhibited behavior, drowsiness; Grade 3: somnolence, bizarre behavior, confusion, amnesia, paranoia; Grade 4: Coma. ${ }^{3}$ PT, Prothrombin Time, also expressed in seconds prolonged < 4; 4-6; > 6, or as INR < I.7; I.7-2.3; > 2.3. US: UltraSound. 


\section{Ultrasound and Doppler ultrasound features of spleno- renalshunts}

The SRS detection was based on the following features:

Evidence of tortuous, inferiorly directed vessels from the splenic hilum to the left kidney (when dealing with the planes of orientation, i.e., coronal, horizontal, and sagittal, they were primarily seen on the coronal ones);

\section{Splenofugal blood flow [31];}

Dilatation of the left renal vein with blood flow phasic and at high velocity $(\geq 20 \mathrm{~cm} / \mathrm{sec})$;

Marked increase of the spleen volume (see below);

Dilatation of the splenic vein diameter $(\geq 5 \mathrm{~mm})$ with blood flow phasic and at high velocity (SVFV $\geq 15 \mathrm{~cm} / \mathrm{sec}$ );

\section{Hepatofugal blood flow in the portal vein}

Spleen Longitudinal Diameter (SLD) was performed by postero-lateral scanning. The Maximum Length $(\mathrm{ML}$, the optically greatest overall longitudinal dimension obtained from one of the two poles) and the Cranio-Caudal Length (CCL, the optically maximal transversal dimension intercepting one of the two poles) were measured; the resulting values were then averaged, since the two measurements do not always coincide. SLD, (ML + $\mathrm{CCL} / 2$ ), $\geq 150 \mathrm{~mm}$ made the spleen be considered definitely enlarged, being this parameter best correlated to its size.

\section{Statistics}

BMI, SVFV and Child-Pugh's classification values, not normally distributed (Shapiro-Wilk test, $\mathrm{p}=0.001,0.001$ and 0.01), were expressed as median (range). HE, EV and ascites grades were considered ordinals and managed in the same way. The difference in medians was assessed by the Kolmogorov-Smirnov (K-S) Two-Sample test. Age data, derived from a normally distributed population (Shapiro-Wilk test, $\mathrm{p}=0.44$ ), were articulated as mean plus SD and 95\% confidence intervals (CI); the difference of means was evaluated by Two-Sample t test. The TwoWay Tables cross-tabulated one or more than one categorical row variables with one categorical column variable and the significance was set by the Pearson Chi-square. When cross-tabulation was stratified for another dichotomous variable the Mantel-Haenszel Chi-square was carried out. The correlation between Child-Pugh's scores and SVFV values was determined by the Spearman's rho. To make prediction the logistic regression (Enter method) was adopted, selecting as independent variable BMI values as well as the Child-Pugh classification and as dependent variable SRS presence/absence; HE and ascites severity were not tested in the context of the previous independent variables to avoid multi-collinearity (situation in which the predictors are correlated to each other to some degree). To assess the independent effect of a quantitative variable, i.e., SFV, on the prediction of the ascites, HE and EV grades the linear regression analysis (least squares) was used, evaluating the standardized coefficient beta $(\beta)$. The Factor Analysis was applied to detect the structure in the relationships among variables selecting a subset of variables, which have the highest correlations with the principal component factors. The Cattel Scree plot, with relative eigenvalues, was performed to screen the real factors. Extraction of the main components amounted to a variance maximizing (varimax) rotation of the original variable space. The critical value was calculated by the formula: doubling the Pearson's correlation coefficient for $1 \%$ level of significance (5.152)/square root of patients minus 2, i.e., 0.579. The concordance correlation coefficient $\left(\rho_{c}\right)$, which measures precision and accuracy, was adopted to evaluate the degree of pair observations at US. The power of our sample size was calculated by the non-equality of proportion with a type I error, alpha, of 0.05. Statistical analysis was performed operating on Systat 12 (Richmond, CA, USA) and MedCalc Version $11^{\circledast}$ (Frank Schoonjans) software packages.

\section{Results}

In order to allow readers to gauge how well the study findings apply to their patients (external validity) we stress that eighty one patients, divided into two groups of, i.e., those with and without SRS, were well balanced in respect to severity of disease $(p=0.51$, Table 2$)$, gender $(p=0.76)$ and age $(\mathrm{p}=0.98)$. The median score of $\mathrm{W}-\mathrm{H}$ criteria in

Table 2: Spleno-renal shunts distribution through liver cirrhosis severity.

\begin{tabular}{llll}
\hline CHILD Score & SRS Absent & SRS Present & Total \\
\hline 5 & 9 & 1 & 10 \\
\hline 6 & 13 & 2 & 15 \\
\hline 7 & 16 & 6 & 22 \\
\hline 8 & 13 & 1 & 14 \\
\hline 10 & 7 & 3 & 10 \\
\hline 11 & 4 & 0 & 4 \\
\hline 12 & 3 & 1 & 4 \\
\hline Total & 1 & 1 & 81 \\
\hline
\end{tabular}

SRS, Spleno-renal Shunts; Pearson Chi-square, $\mathrm{p}=0.51$. 
patients with and without SRS was similar, i.e., $0(0-2)$ versus $0(0-3), p=0.67$. The median value of SVFV in patients without SRS was significantly inferior to that of patients with SRS, i.e., $13 \mathrm{~cm} / \mathrm{sec}$ [6-18] versus $21 \mathrm{~cm} / \mathrm{sec}$ [17 - 24], $\mathrm{p}<0.0001$.

\section{Prevalence}

The prevalence of SRS in our patients, screened at DUS, was exactly $18.5 \%$ without any difference concerning the etiology of liver cirrhosis (HCV versus non-HCV, Pearson Chi-square $\mathrm{p}=0.870)$. Ascites, LEV and HE were present in $28.3 \%, 38.3 \%$ and $24.7 \%$ of our population, respectively. By far the largest percentage of LEV was in patients without SRS (Pearson Chi-square, $\mathrm{p}=0.005$, Table 3 ). In contrast, the frequency of HE severity and ascites was overlapping (Tables 4 and 5). The prevalence of HCC in patients with SRS was superior to that of patients without it (Pearson Chi-square, $\mathrm{p}=0.006$, Table 6), also after adjustment for liver decompensation (Mantel-Haenszel Chi-square, $\mathrm{p}=0.024)$. HCC, equally detected on the basis of the gender (Pearson Chi-square, $p=0.436$ ), counted for a part $(8.6 \%)$ of our cohort according to the expected incidence.

\section{Association}

The correlation of the studied DUS parameter, i.e., SVFV, to the degree of liver failure, weighed by Child-Pugh's scores was inversely significant ( $\mathrm{rho}=-0.430, \mathrm{p}=0.001)$.

Interestingly, a hidden correlation was found among the SLD, the SVFV and the BMI, independently from age of patients and perhaps of the illness, Figure 1.

\section{Prediction}

The estimates of Child-Pugh's classification and BMI values in predicting the SRS presence gave the following results: $\mathrm{OR}=1.145,95 \% \mathrm{CI}=0.77-1.51, \mathrm{p}=0.66$ and $\mathrm{OR}$ $=1.84,95 \% \mathrm{CI}=1.28-2.64, \mathrm{p}=0.001$, respectively. Lower SVFV values predicted more advanced ascites, HE and EV grades $(\beta=-0.29, \mathrm{p}=0.009 ; \beta=-0.37, \mathrm{p}=0.001$ and $\beta=$ $-0.41, \mathrm{p}=0.0001$, respectively).

Table 3: Spleno-renal shunts distribution through large esofageal varices presence.

\begin{tabular}{cccc}
\hline LEV & Absent & Present & Total \\
\hline SRS Absent & 36 & 30 & 66 \\
SRS Present & 14 & 1 & 15 \\
Total & 50 & 31 & 81 \\
\hline
\end{tabular}

LEV, Large Esofageal Varices; SRS, Spleno-Renal Shunts; Pearson Chisquare, $\mathrm{p}=0.005$.
Table 4: Spleno-renal shunts distribution through hepatic encephalopathy severity.

\begin{tabular}{cccccc}
\hline HE Grade & Absent or Minimal & I & $\mathbf{2}$ & $\mathbf{3}$ & Total \\
\hline SRS Absent & 50 & 9 & 6 & 1 & 66 \\
SRS Present & 10 & 4 & 1 & 0 & 15 \\
Total & 60 & 13 & 7 & 1 & 81 \\
\hline
\end{tabular}

SRS, Spleno-Renal Shunts; HE, Hepatic Encephalopathy; Pearson Chisquare, $p=0.63$.

\section{Accuracy}

The agreement of US paired observations ranked high, i.e., $\rho_{c}=0.90$.

\section{Power analysis}

When analysing the impact of the sample size regarding the HCC presence through the SRS distribution, the power was $74 \%$ with a type I error of 0.05 .

\section{Discussion}

The essential findings we provide in this study are briefly i) greater occurrence of HCC in patients with SRS than in those without them; ii) clear prediction of BMI towards the SRS presence; iii) detection of fewer LEV in patients presenting SRS; iiii) same prevalence of ascites and HE presence in patients with and without SRS. Although knowledge regarding mechanisms involved in the pathogenesis of PH has taken unprecedented levels, nevertheless many prognostic aspects still remain to be elucidated. If one considers that knowing them could help better manage cirrhotic patients, it would then be justifiable to speculate whether physicians could predict the history of liver cirrhosis on the basis of the spontaneous SRS presence by some means. This study aimed at partially answering this question. The body of present knowledge [9] is in favour of the fact that the presence of portosystemic collaterals identifies cirrhotics with a less favourable clinical course. Our data only partially agree with this conclusion. In fact, we provide evidence for a low risk of LEV and a quite similar prevalence of $\mathrm{HE}$ and ascites in patients with SRS. However, the procrastination of haemorrhagic risk from LEV in SRS patients would be only temporary, because the basal critical PH identifies a more severe grade

Table 5: Spleno-renal shunts distribution through ascites severity.

\begin{tabular}{ccccc}
\hline Ascites & Absent & Grade I & Grade 2 & Total \\
\hline SRS Absent & 46 & 13 & 7 & 66 \\
SRS Present & 12 & 2 & 1 & 15 \\
Total & 58 & 15 & 8 & 81 \\
\hline
\end{tabular}

SRS, Spleno-Renal Shunts; Pearson Chi-square, $\mathrm{p}=0.73$. 
Table 6: Spleno-renal shunts distribution through hepatocellular carcinoma presence

\begin{tabular}{cccc}
\hline HCC & Absent & Present & Total \\
\hline SRS Absent & 63 & 3 & 66 \\
SRS Present & 11 & 4 & 15 \\
Total & 74 & 7 & 81 \\
\hline
\end{tabular}

SRS, Spleno-Renal Shunts; HCC, HepatoCellular Carcinoma; Pearson Chi-square, $p=0.006$.

of liver cirrhosis, i.e., very high and long-lasting sinusoidal resistance.

Some of our results are expected if the following is examined. A recent study comparing Endoscopic Sclerotherapy (ES) with distal surgical portosystemic shunts in the prevention of recurrent variceal bleeding, has showed that both these interventions achieved a positive effect on variceal rebleeding [32]. A switch to decompressive shunt procedures is mandatory in case of ES or Transjugular Intrahepatic Portosystemic Shunt (TIPS) insertion fails to

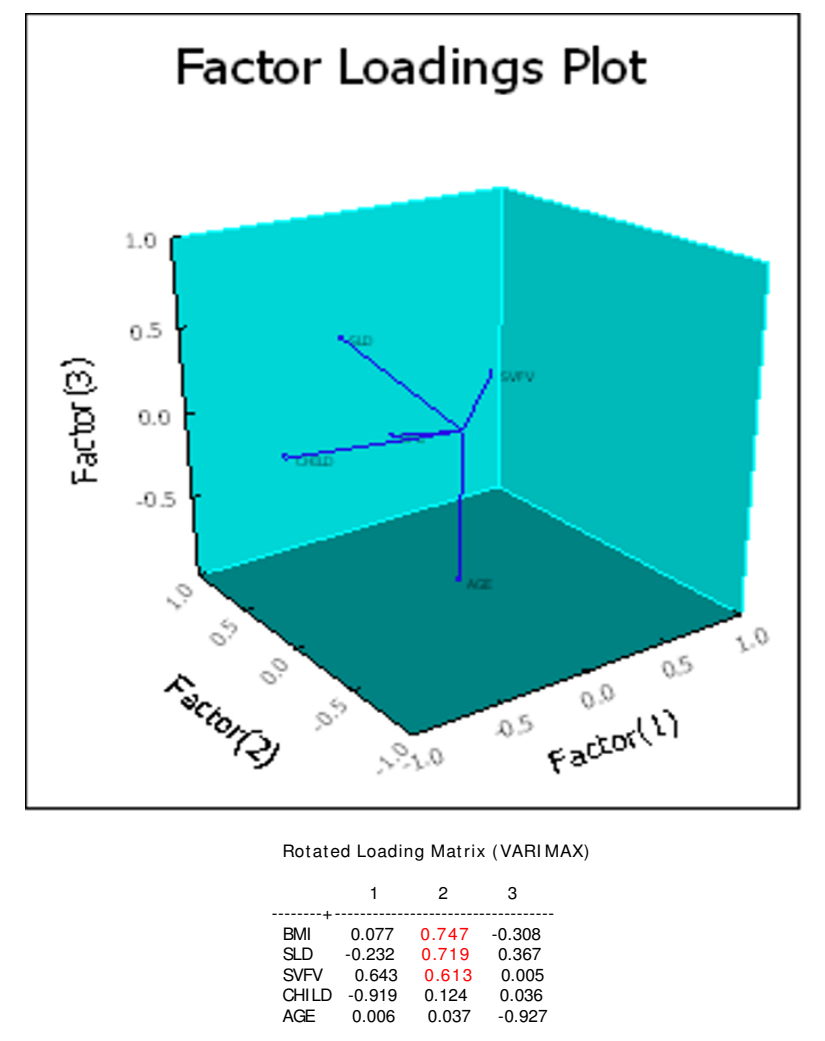

\section{Figure I}

Factor Loadings Plot. Body Mass Index, BMI; Spleen Longitudinal Diameter, SLD; Splenic Vein Flow velocity, SVFV. The highest loading is on Factor 2, where three variables are strictly correlated. control recurrent variceal hemorrhage [33]. Selective shunts are placed surgically to manage post-transplant portal vein stenosis/thrombosis. In contrast, preexisting spontaneous portosystemic shunts increase the risk of post-transplantation portal vein thrombosis [34] and hamper graft survival [35]. Furthermore, SRS main disadvantage is decreased perfusion of the liver with eventually reduction in liver volume and related function [36]. Nevertheless, surgeons are convinced that the creation of peripheral portosystemic shunt still has a role in the treatment of some patients with $\mathrm{PH}$ on the basis that longterm blood flow in the portal vein was not severely reduced after this intervention [37]. Finally, a meta-analysis of individual patient data has provided further evidence that TIPS significantly impacts only on those patients with the extreme grade of ascites [38], although it worsens encephalopathy [39]. The novelty of our study relies on the evidence of an interesting percentage of patients with SRS in which HCC has been detected, mainly taking into consideration that HCC prevalence in our population was lower than that found among Japanese patients (confined to HCV infection), obtained summarizing four representative studies [40], which was $16 \%$ $(212 / 1339)$. But, how can we explain this major occurrence of HCC in them? We emphasize that HCC, the third leading cause of cancer mortality worldwide, is a highly vascular tumor that expresses VEGF [41]. An up-to-date study provides further evidence that obesity increases HCC risk and that this factor may explain a relevant proportion of cases among subjects in absence of $\mathrm{HBV} / \mathrm{HCV}$ infection, probably NASH-mediated [42]. Interestingly, patients suffering from NASH, a further expression of the metabolic syndrome, express high serum concentrations of VEGF [43]. Finally, portal VEGF was significantly higher than systemic VEGF, and expressions of VEGF and hepatocyte growth factor in the liver, spleen and intestine were also up-regulated during liver regeneration [44].

In term of clinical advantage, which take-home lesson could be drawn from our data? Physicians should try to strike a balance between pprogressive nutrition deficiencies as well as muscle wasting, universal problems in patients suffering from liver cirrhosis, and a moderately hypocaloric diet to avoid this eventual risk factor.

As it is repeatedly emphasized, PH remains a partiallyclarified phenomenon and so does the collaterals presence. Now, the VEGF-dependent angiogenesis is considered being crucial in determining SRS [2]. A fascinating hypothesis suggests that portosystemic shunts may mimic an Arterio-Venous Fistula (A-VF [45]), in which the highpressure portal blood relays with the lower pressure systemic venous circulation. Although these collaterals decompress the portal circulation, the increased cardiac output enhances portal blood flow and tends to counter- 
act the portal hypotensive effect of the portosystemic shunt. As portal blood flow grows, collateral blood flow multiplies and is nearly totally shunted in the systemic circulation. Ultimately, high-output cardiac failure occurs leading to cirrhotic cardiomyopathy. Actually, researchers demonstrated an over-expression of VEGF in a rabbit A-VF model [46].

Coming back to the HE detection in both patients with and without SRS, what does support this finding? In contrast with the current opinion, a recent study in patients with cirrhosis has failed to find a relationship between EEG alterations and the presence per se of a patent paraumbilical vein, very common collateral with the same significance of SRS [7]. Comparing angiographically patients evidencing LSS and HE with cases characterized by PH but no HE, Takashi et al. concluded that small SRS were not associated with HE [47]. On the other hand, some patients with spontaneous shunts, who had undergone shunt reduction by a radiological approach [48], obtained an amelioration of HE. In conclusion, SRS could only provide an explanation for the refractoriness of HE. Continuing on the subject, the lack of association between liver cirrhosis severity and SRS, found in our series, is confirmed by the subsequent observations. Following-up consecutive patients with bleeding varices and LSS, after devascularization with or without esophageal transaction, the postoperative survival rate of patients with the LSS was significantly lower than that of patients without the LSS and preoperative variables concerning hepatic reserve failed to show significant predictability [16]. Other data challenge this finding [9]. Discussing the possible limitations of the present study we have to pinpoint that the sample size of patients with HCC is small, but it seems to be of sufficient power and adequate to the low $(5 \%)$ incidence of this complication in liver cirrhosis [49]. Moreover, it is correct to evidence that other cuttingedge technology is more specific for the detection of portosystemic collaterals, e.g., MRI [13]. But, any crucial future research directions should consider the utility of DUS particularly when repeated measures are requested beyond liver transplantation settings [34,35]. Last but not least consideration, are we sure that splenomegaly is the consequence of $\mathrm{PH}$ and not one of its causes, mainly taking into consideration the spleno-renal reflex-mediated reduction in vascular conductance [50] that exacerbates sodium and water retention in the kidneys?

\section{Conclusion}

SRS in liver cirrhosis is a feature more neglected in clinical practice than among imaging operators. Forecasting whether the search for SRS would be carried out years down the line is a dodgy business, because this attitude is not popular with physicians even though it could influence the prognostic choices. What will the positive and negative (in respect to the moderately increased costs) effects be, it is necessary to ascertain. Anyway, patients with evidence of SRS are burdened by an enhanced incidence of HCC, mainly if they are overweight or obese. The awareness of this crucial complication should increase in order to bring a survival advantage to those patients.

\section{Abbreviation list}

PH: Portal Hypertension; EV: Esophageal Varices; HE: HepaticEncephalopathy; LSS: Large Spontaneous Shunts; SRS: Spleno-RenalShunts; US: UltraSound; MRI: Magnetic Resonance Imaging; HCC:HepatoCellular Carcinoma; VEGF: Vascular Endothelial Growth Factor; DUS: Doppler UltraSound; TIPS: Transjugular Intrahepatic Portosystemic Shunt; SVFV: Splenic Vein Flow Velocity; LEV: Large EV; ES: Endoscopic Sclerotherapy; W-H: West Haven; ML: Maximum Length; CCL: Cranio-Caudal Length; SLD: Spleen Longitudinal Diameter; $\beta$ : standardized coefficient beta; $\rho_{c}$ : correlationcoefficient.

\section{Competing interests}

The authors declare that they have no competing interests.

\section{Authors' contributions}

TG conceived of the study and drafted the manuscript. CV and CP carried out the clinical investigation. IV participated in the design of the study, and RA and CD helped to draft the manuscript. TM and TG performed ultrasound. TG performed the statistical analysis. CM and LR made endoscopy. All authors read and approved the final manuscript.

\section{References}

I. Blei AT: Portal hypertension and its complications. Curr Opin Gastroenterol 2007, 23:275-282.

2. Fernandez M, Mejias M, Garcia-Pras E, Mendez R, Garcia-Pagan JC, Bosch J: Reversal of portal hypertension and hyperdynamic splanchnic circulation by combined vascular endothelial growth factor and platelet-derived growth factor blockade in rats. Hepatology 2007, 46:1208-1217.

3. Ripoll C, Groszmann RJ, Garcia-Tsao G, Bosch J, Grace N, Burroughs A, Planas R, Escorsell A, Garcia-Pagan JC, Makuch R, Patch D, Matloff DS, Portal Hypertension Collaborative Group: Hepatic venous pressure gradient predicts development of hepatocellular carcinoma independently of severity of cirrhosis. I Hepatol 2009, 50:923-928.

4. Del Piccolo F, Sacerdoti D, Amodio P, Bombonato G, Bolognesi M, Mapelli D, Gatta A: Central nervous system alterations in liver cirrhosis: the role of portal-systemic shunt and portal hypoperfusion. Metab Brain Dis 2002, 17:347-358.

5. De Franchis R, Salerno F: Pathogenesis of ascites and predictors of resistance to therapy. J Gastroenterol Hepatol 2002, 17:S242-247.

6. Primignani $M$, Carpinelli L, Preatoni P, Battaglia G, Carta A, Prada A, Cestari R, Angeli P, Gatta A, Rossi A, Spinzi G, De Franchis R: Natural history of portal hypertensive gastropathy in patients with liver cirrhosis. The New Italian Endoscopic Club for the study and treatment of esophageal varices (NIEC). Gastroenterology 2000, I1 19:181-187.

7. Merli M, Nicolini G, Angeloni S, Gentili F, Attili AF, Riggio O: The natural history of portal hypertensive gastropathy in patients with liver cirrhosis and mild portal hypertension. Am J Gastroenterol 2004, 99:1959-1965. 
8. Mostbeck GH, Wittich GR, Herold C, Vergesslich KA, Walter RM, Frotz S, Sommer G: Hemodynamic significance of the paraumbilical vein in portal hypertension: assessment with duplex US. Radiology 1989, 170:339-342.

9. Zardi EM, Uwechie $V$, Caccavo D, Pellegrino NM, Cacciapaglia F, Di Matteo F, Dobrina A, Laghi V, Afeltra A: Portosystemic shunts in a large cohort of patients with liver cirrhosis: detection rate and clinical relevance. J Gastroenterol 2009, 44:76-83.

10. von Herbay A, Frieling T, Haussinger D: Color Doppler sonographic evaluation of spontaneous portosystemic shunts and inversion of portal venous flow in patients with cirrhosis. Clin Ultrasound 2000, 28:332-339.

II. Bresci G, Gambardella L, Parisi G, Federici G, Bertini M, Rindi G, Metrangolo S, Tumino E, Bertoni M, Cagno MC, Capria A: Colonic disease in cirrhotic patients with portal hypertension: an endoscopic and clinical evaluation. J Clin Gastroenterol 1998, 26:222-227.

12. Riggio O, Efrati C, Catalano C, Pediconi F, Mecarelli O, Accornero N Nicolao F, Angeloni S, Masini A, Ridola L, Attili AF, Merli M: High prevalence of spontaneous portal-systemic shunts in persistent hepatic encephalopathy: a case-control study. Hepatology 2005, 42: II58-II65.

13. Kim M, Mitchell DG, Ito K: Portosystemic collaterals of the upper abdomen: review of anatomy and demonstration on MR imaging. Abdom Imaging 2000, 25:462-470.

14. Kashiwagi T, Kimura K, Kozuka T, Sato N, Kamada T, Mitsutani N, Koizumi T: Portosystemic collaterals in portal hypertension: visualization by using blood-pool SPECT imaging. Am J Roentgenol 1989, I 53:28I-285.

15. Azuma M, Kashiwagi T, Nagasawa M, Ito Y, Naito M, Ishibashi K, Katayama K: Evaluation of portosystemic collaterals by SPECT imaging after endoscopic variceal sclerotherapy: usefulness for predicting recurrence. J Nucl Med 2000, 41:600-604.

16. Nakamura R, Hirose A, Nakasato T, Yoshioka K, Sasaki M, Tamakawa $Y$, Sasaki A: Detection of paraumbilical or splenorenal shunts by intra-arterial-DSA in bleeding varices: another risk factor? Radiat Med 1998, 16:34I-345.

17. Kashiwagi T, Kimura K, Suematsu T, Shichiri M, Kamada T, Abe H: Dynamic studies on portal haemodynamics by scintiphotosplenoportography: flow patterns of portal circulation. Gut 1980, 21:57-62.

18. Smith Laing G, Camilo ME, Dick R, Sherlock S: Percutaneous transhepatic portography in the assessment of portal hypertension: clinical correlation and comparison of radiographic techniques. Gastroenterology 1980, 78:197-205.

19. Martínez-Noguera A, Montserrat E, Torrubia S, Villalba J: Doppler in hepatic cirrhosis and chronic hepatitis. Semin Ultrasound CT MR 2002, 23:19-36.

20. Robinson KA, Middleton WD, Al-Sukaiti R, Teefey SA, Dahiya N: Doppler sonography of portal hypertension. Ultrasound $Q$ 2009, 25:3-13.

21. Annet L, Materne R, Danse E, Jamart J, Horsmans Y, Van Beers BE: Hepatic flow parameters measured with Mmirroring $R$ imaging and Doppler US: correlations with degree of cirrhosis and portal hypertension. Radiology 2003, 229:409-4I4.

22. Perisic M, llic-Mostic T, Stojkovic M, Culafic D, Sarenac R: Doppler hemodynamic study in portal hypertension and hepatic encephalopathy. Hepatogastroenterology 2005, 52: I56-160.

23. Yin XY, Lu MD, Huang JF, Xie XY, Liang L): Color Doppler velocity profile assessment of portal hemodynamics in cirrhotic patients with portal hypertension: correlation with esophageal variceal bleeding. J Clin Ultrasound 200I, 29:7-13.

24. Luo JC, Hwang SJ, Chang FY, Chu CW, Lai CR, Wang YJ, Lee PC, Tsay SH, Lee SD: Simple blood tests can predict compensated liver cirrhosis in patients with chronic hepatitis C. Hepatogastroenterology 2002, 49:478-481.

25. Beppu K, Inokuchi K, Koyanagi N, Nakayama S, Sakata H, Kitano S, Kobayashi M: Prediction of variceal hemorrhage by esophageal endoscopy. Gastrointest Endosc 1981, 27:213-2।8.

26. Gooding GA, Cummings SR: Sonographic detection of ascites in liver disease. J Ultrasound Med 1984, 3:69-72

27. Conn HO, Liebertahl MM: The hepatic coma syndromes and lactulose. Williams and Wilkins, Baltimore; 1979:I-I2I.

28. Citro V, Milan G, Tripodi FS, Gennari A, Sorrentino P, Gallotta G, Postiglione A, Tarantino G: Mental status impairment in patients with West Haven grade zero hepatic encephalopathy: the role of HCV infection. J Gastroenterol 2007, 42:79-82.

29. Tarantino G, Gentile A, Capone D, Basile V, Tarantino M, Di Minno $\mathrm{MN}$, Cuocolo A, Conca P: Does protracted antiviral therapy impact on HCV-related liver cirrhosis progression? World J Gastroenterol 2007, 13:4903-4908.

30. Planas R, Balleste B, Alvarez MA, Rivera M, Montoliu S, Galeras JA Santos J, Coll S, Morillas RM, Solà R: Natural history of decompensated hepatitis $C$ virus-related cirrhosis. A study of $\mathbf{2 0 0}$ patients. J Hepatol 2004, 40:823-830.

31. Von Herbay A, Frieling T, Haussinger D: Color Doppler sonographic evaluation of spontaneous portosystemic shunts and inversion of portal venous flow in patients with cirrhosis. J Clin Ultrasound 2000, 28:332-339.

32. Santambrogio R, Opocher E, Costa M, Bruno S, Ceretti AP, Spina GP: Natural history of a randomized trial comparing distal spleno-renal shunt with endoscopic sclerotherapy in the prevention of variceal rebleeding: a lesson from the past. World J Gastroenterol 2006, I 2:633I-6338.

33. Khan S, Tudur Smith C, Williamson P, Sutton R: Portosystemic shunts versus endoscopic therapy for variceal rebleeding in patients with cirrhosis. Cochrane Database Syst Rev 2006, 4:CD000553

34. Braun MM, Bar-Nathan N, Shaharabani E, Aizner S, Tur-Kaspa R, Belenky A, Mor E, Ben-Ari ZZ: Postshunt hepatic encephalopathy in liver transplant recipients. Transplantation 2009, 87:734-739.

35. Nakano T, Lai CY, Goto S, Hsu LW, Huang TL, Chen TY, Tsang LC, Chen CL, Cheng YF: Significance of portosystemic shunt on graft survival in liver transplantation: a rat model. Transplant Proc 2008, 40:25I5-25I6.

36. Sato Y, Mitsuma C, Iwaya A, Kurosaki I, Shirai Y, Hatakeyama K: Resection of spleno-renal shunt resulting in enhanced liver volume in a patient with congenital portosystemic shunt concomitant with early gastric cancer. Review of Japanese cases. Dig Surg 200I, I 8:74-78.

37. Král V, Klein J, Havlik R, Vomácka J, Utikal P, Vrba R: Peripheral portosystemic shunt and its selectivity changes measured on duplex ultrasound. Hepatogastroenterology 2005, 52:|49-15|.

38. Salerno F, Cammà C, Enea M, Rössle M, Wong F: Transjugular intrahepatic portosystemic shunt for refractory ascites: a meta-analysis of individual patient data. Gastroenterology 2007 133:825-834.

39. Albillos A, Bañares R, González M, Catalina MV, Molinero LM: A meta-analysis of transjugular intrahepatic portosystemic shunt versus paracentesis for refractory ascites. I Hepatol 2005, 43:990-996.

40. Umemura T, Ichijo T, Yoshizawa K, Tanaka E, Kiyosawa K: Epidemiology of hepatocellular carcinoma in Japan. J Gastroenterol 2009, 44:102-107.

4I. Schoenleber SJ, Kurtz DM, Talwalkar JA, Roberts LR, Gores G] Prognostic role of vascular endothelial growth factor in hepatocellular carcinoma: systematic review and meta-analysis. Br J Cancer 2009, 1 00:1385-1392.

42. Polesel J, Zucchetto A, Montella M, Dal Maso L, Crispo A, La Vecchia $C$, Serraino D, Franceschi S, Talamini R: The impact of obesity and diabetes mellitus on the risk of hepatocellular carcinoma. Ann Oncol 2009, 20:353-357.

43. Tarantino G, Conca P, Pasanisi F, Ariello M, Mastrolia M, Arena A Tarantino M, Scopacasa F, Vecchione R: Could inflammatory markers help diagnose nonalcoholic steatohepatitis? Eur ] Gastroenterol Hepato 2009, $21: 504-5$ II.

44. Yamamoto C, Yagi S, Hori T, lida T, Taniguchi K, Isaji S, Uemoto S: Significance of Portal Venous VEGF During Liver Regeneration After Hepatectomy. J Surg Res 2008 in press.

45. Wattanasirichaigoon S, Gordon FD, Resnick RH: Hyperdynamic circulation in portal hypertension: a comparative model of arterio-venous fistula. Med Hypotheses 2000, 55:77-87.

46. Sho E, Komatsu M, Sho M, Nanjo H, Singh TM, Xu C, Masuda $H$, Zarins CK: High flow drives vascular endothelial cell proliferation during flow-induced arterial remodeling associated with the expression of vascular endothelial growth factor. Exp Mol Pathol 2003, 75: I-II.

47. Takashi M, Igarashi M, Hino S, Takayasu K, Goto N, Musha H, Ohnishi $\mathrm{K}$, Okuda K: Portal hemodynamics in chronic portal-systemic 
encephalopathy. Angiographic study in seven cases. J Hepatol 1985, I:467-476.

48. Kato T, Uematsu T, Nishigaki Y, Sugihara J, Tomita E, Moriwaki H: Therapeutic effect of balloon-occluded retrograde transvenous obliteration on portal-systemic encephalopathy in patients with liver cirrhosis. Int Med 200I, 40:677-678.

49. Kiyosawa K: Trend of liver cirrhosis as precancerous lesions. Hepatol Res 2002, 24:40-45.

50. Hamza SM, Kaufman S: Role of spleen in integrated control of splanchnic vascular tone: physiology and pathophysiology. Can J Physiol Pharmacol 2009, 87: I-7.

\section{Pre-publication history}

The pre-publication history for this paper can be accessed here:

http://www.biomedcentral.com/1471-230X/9/89/pre

pub

Publish with Bio Med Central and every scientist can read your work free of charge

"BioMed Central will be the most significant development for disseminating the results of biomedical research in our lifetime."

Sir Paul Nurse, Cancer Research UK

Your research papers will be:

- available free of charge to the entire biomedical community

- peer reviewed and published immediately upon acceptance

- cited in PubMed and archived on PubMed Central

- yours - you keep the copyright

Submit your manuscript here:

http://www.biomedcentral.com/info/publishing_adv.asp 